\title{
An fMRI Investigation of Moral Cognition in Healthcare Decision Making
}

\author{
Lisa J. Smith \\ Flinders University
}

\author{
Paul Anand \\ The Open University and Oxford University
}

\author{
Abdelmalek Benattayallah \\ University of Exeter
}

\author{
Timothy L. Hodgson \\ University of Lincoln
}

\begin{abstract}
This study used fMRI to investigate the neural substrates of moral cognition in health resource allocation decision problems. In particular, it investigated the cognitive and emotional processes that underpin utilitarian approaches to health care rationing such as Quality Adjusted Life Years (QALYs). Participants viewed hypothetical medical and nonmedical resource allocation scenarios which described equal or unequal allocation of resources to different groups. In addition, participants were assigned to 1 of 2 treatments in which they either did or did not receive advanced instructions about the principles of utilitarianism. In all cases, participants were asked to judged the proposed allocations as "fair" or "unfair." More brain activity was observed within the superior parietal lobe, angular gyrus, middle temporal gyrus, and bilateral caudate nucleus when participants viewed scenarios depicting equal divisions of resources. Conversely, unequal resource divisions were associated with more activity in the inferior frontal gyrus and insula cortex. Furthermore, instructions about the principles of utilitarianism led to significant activation differences within the inferior frontal gyrus and the middle frontal gyrus. Significant differences in activity were also found within the inferior frontal cortex and anterior insula between medical and nonmedical scenarios. The implications for cognitive control mechanisms and the cognitive and neural bases of utilitarian ethical judgment are discussed.
\end{abstract}

Keywords: equality, fMRI, health care, QALY, utilitarianism

How to divide limited public resources for health care fairly among a population is a major moral and political issue in all countries around the world. Research has shown that people are attentive to perceived breaches of individual rights to treatment when asked to make health

Lisa J. Smith, School of Psychology, Flinders University; Paul Anand, Department of Economics, Faculty of Social Sciences, The Open University, and Health Economics Research Centre, Nuffield Department of Public Health, Oxford University; Abdelmalek Benattayallah, Exeter Magnetic Resonance Imaging Research Centre, University of Exeter; Timothy L. Hodgson, School of Psychology, University of Lincoln.

Correspondence concerning this article should be addressed to Timothy L. Hodgson, School of Psychology, University of Lincoln, Brayford Campus, Lincoln LN6 7TS, United Kingdom. E-mail: tlhodgson@lincoln.ac.uk care rationing decisions for themselves (Anand \& Walloo, 2000). In contrast, utilitarian perspectives argue that the 'rational' way in health care resource allocation is not to heavily weight universal rights, but to allocate resources which maximize the widest public good.

Neuroscientific research into moral cognition has emphasized the importance of both emotion and cognition in forming choices, although to date techniques of neuroscience and neuropsychology have not been applied to understanding health care rationing decision making (Ciaramelli et al., 2007; Koenigs et al., 2007; Moll, de Oliveira-Souza \& Eslinger, 2003; Moll, Eslinger \& de Oliveira-Souza, 2001; Moll et al., 2002; Moll \& de Oliveira-Souza, 2007; Young \& Koenigs, 2007; Koenigs et al., 2007). As well as being of interest in its own right, the question 
of the extent to which brain areas involved in emotion and cognitive control contribute to such decision making might potentially inform the reason why people make the choices they do in these context. In the present study, we use functional MRI (fMRI) to examine how brain regions involved in decision making, cognition, and emotion are recruited in health care rationing decision making.

Utilitarianism, or more specifically 'Act Utilitarianism,' is a moral framework which proposes that one should choose actions that yield at least as high a utility as the sum total of utilities of any other alternative act (Bentham, 1789). This moral framework has been highly influential within economics and has given rise to the use of a QALY (Quality Adjusted Life Year) maximization rule by economists working on issues to do with health. Quality of life factors include loss of function and mobility and the enjoyment one can derive from life after resources have been allocated (Cubbon, 1991). In essence, the expected QALY benefit of a medical intervention is the measure of its average value and the set of interventions which should be funded according to utilitarianism is that which maximizes the total gain in QALYs by a society (Appleby, Devlin, \& Parkin, 2007).

However, utilitarian resource allocation has been criticized by philosophers concerned about justice and distributional aspects of fairness within a "Rawlsian" perspective (Rawls, 1971). According to Rawls each person has an equal right to the most extensive basic liberty (greatest liberty principle) and inequalities are only permitted if they benefit all persons. For something to be just or fair it must not violate principles of liberty or equality. Utilitarianism permits distributional inequalities if they serve the general interest in contrast to the egalitarian stance of justice outlined by Rawls.

QALY maximization is perceived by some as condemning people who are not 'cost-effective' to society if their care does not produce enough medical benefit. If all other things are equal, the QALY approach could prioritize the health of someone who already has a high health status. A person who is disabled will be viewed as having a poorer long term quality of life and so any QALY score they obtain for treatment will be inevitably lower than that of a healthy per- son. Some philosophers (e.g., Harris, 1987, 1995, 2005) object to the use of QALYs on this basis, arguing that it puts such chronically ill or disabled persons in a sort of 'double jeopardy.' Not only do they suffer a misfortune by becoming disabled or otherwise disadvantaged in the first instance, they are as a result given a lower priority in their health care. Empirical research also demonstrates that people do not make decisions consistent with QALYs (Anand \& Walloo, 2000). It has been suggested that the QALY approach fails to account for real choice behavior because it does not reflect the moral beliefs held by ordinary members of the public who often see the results of QALY decision making frameworks as violating claims based on human rights or social contracts (Anand \& Walloo, 2000).

In contrast to moral decision-making frameworks such as utilitarianism and QALYs which emphasizes rational calculation, research in cognitive neuroscience emphasizes the contribution of emotion to decision making in real social situations (e.g., Bechara \& Damasio, 2005). Analysis of brain activity via fMRI during economic "games" and moral decision making tasks reveals interacting networks of activation within prefrontal and subcortical structures associated with value formation, cognition and emotion. For example, Sanfey, Rilling, Aronson, Nystrom, and Cohen (2003) used fMRI to measure brain activity in the 'ultimatum game,' in which two players are given the opportunity to split a sum of money: one player acts as the proposer and the responder must either accept or reject the offer. Traditional economic theory would predict that the proposer should offer the smallest sum of money possible and that the responder should accept on the premise that some money is preferable to none. The bilateral anterior insula, dorsolateral prefrontal cortex (DLPFC), and anterior cingulate cortex generate greater activation in responder's brains for unfair compared with fair offers. The insula in particular has been associated with the experience of disgust, an emotion that has been conjectured to play an important role in moral decision making (Haidt, Rozin, McCauley, \& Imada, 1997; Moll et al., 2005).

Greene, Nystrom, Engell, Darley, and Cohen (2004) used fMRI to study variants of the classic "trolley car" problem. In the original version of this problem, participants were asked to 
make a hypothetical decision to save 5 lives in a runaway trolley car at the cost of sacrificing one individual by pulling a lever to divert the train onto an alternative track. In the "bridge variant," the 5 lives can only be saved by pushing a fat man off a bridge into the path of the train. Green et al. found that brain areas associated with emotion and cognition exhibited higher levels of activation for personal moral judgments (e.g., the bridge variant problem) versus impersonal moral judgments. More difficult personal moral judgments showed increasing activity in the DLPFC and the ACC. By contrast, increased activity was found in the DLPFC only for impersonal moral judgments which resembled utilitarian decisions.

Based on these findings, Green and colleagues argued that difficult personal moral judgments involve a conflict between cognitive and emotional processes. They proposed a 'dual process' model of moral cognition in which personal moral judgments are driven by social emotional processes and impersonal moral judgments are more cognitive in nature.

Subsequent work has also suggested that utilitarian moral judgments are driven by controlled cognitive processes, whereas nonutilitarian judgments are driven by automatic emotional responses (Greene, 2007). Greene, Morelli, Lowenberg, Nystrom, and Cohen (2008) presented participants with personal and impersonal moral dilemmas either under cognitive load (digit search task) or a control condition (no cognitive load). They found that utilitarian judgments were significantly slower than nonutilitarian judgments under cognitive load. Greene and colleagues argue that this provides direct evidence for their view that utilitarian judgments are driven by controlled cognitive processes, whereas nonutilitarian judgments are driven by automatic (more emotional/affective) reasoning processes. In other research, it has been found that patients with lesions of the ventro-medial prefrontal cortex (VMPFC) produced an abnormally high rate of utilitarian judgments in cases which required the individual to overcome an emotional response to the direct harming of others (Koenigs et al., 2007). These patients also exhibited more normal judgments for impersonal situations, suggesting that the VMPFC is a crucial brain structure for the mediation of moral judgments that involve emotionally salient actions.
Based on these previous investigations of non-health care decision making scenarios, we hypothesized that when people form decisions based on QALY maximization principles, increased engagement of neural centers responsible for cognitive control and reasoning should be observed (e.g., DLPFC). Our rationale is that when individuals make a utilitarian judgment, it is likely to require the suppression of an immediate and powerful emotional response to the perceived intergroup inequality and breach of human rights to health care. Conversely, it is hypothesized that when people make decisions which go against QALY maximization, and choose not to allocate resources to those with greater expected utilities, then this will be associated with enhanced neural responses associated with emotions (e.g., anterior insula). Moreover, as health care rationing might be considered a particularly personal moral dilemma, enhanced activity in emotional centers and more Rawlsian or rights-based decision making should be seen in such problems. This study investigated these hypotheses using fMRI scanning while participants considered a series of hypothetical resource allocation split scenarios. In each scenario, they indicated with a button press whether they considered them to be "fair" or "unfair."

\section{Method}

\section{Participants}

Thirty participants were recruited from advertisements placed within the University of Exeter campus and from internal mailing lists. Participants were paid 10 pounds and were aged between 19 and 36 years $(M=26 ; 5$ male, 25 female). Ethical approval was obtained from the University of Exeter School of Psychology ethics committee. Exclusion criteria specified were major medical illness (e.g., congenital abnormalities, heart problems, cancer) neurological disorders (e.g., Seizures, Head Injury), or metal fragments/implants which make MRI contraindicated.

Half the participants received instructions about utilitarian/QALY decision making frameworks as part of the information and consent 
procedure $^{1}$ (QALY Instructed participants). The other group received no advanced briefing on utilitarian decision making (Uninstructed group).

\section{Task and Materials}

The experimental task involved the presentation of 60 stimulus slides which required participants to make a button press response to indicate whether they considered a resource allocation/'split' of money between two social groups (defined by QALY relevant factors) to be either fair or unfair. A control task involved judgments about how correctly (or "fairly") a horizontal bar indicated a stated quantitative split of a commodity. Stimuli used in the fMRI study were designed using Microsoft PowerPoint and then exported into "jpg" format at $1024 \times 768$ pixel resolution. Each scenario screen was displayed for up to 20 seconds, followed by presentation of a fixation cross for a variable period of 3 to 12 seconds. Participants viewed the stimuli in the scanner via a mirror mounted on the head coil and a back projection screen located at the foot of the scanner. Responses were made using a fiber optic response key box. Custom written C-software was used to display the image, record responses and control stimulus timing via a laptop PC in the scanner control room.

A horizontal bar in the stimulus screen was designed to graphically represent the hypothetical allocation of resources (always a split of $£ 10$ million) to two groups who differed on QALY relevant factors (i.e., married versus non married, children versus no children, high versus low income, and old versus young). The stimulus set of 60 slides contained, for every participant, 20 Medical scenarios, 20 Nonmedical scenarios, and 20 Control scenarios. The Medical scenarios were organized into two groups: Group A (depression, diabetes, liver disease, dementia, cancer) and Group B (HIV, asthma, epilepsy, influenza, heart disease). The Nonmedical scenarios comprised Group A (basketball lessons, super high speed internet, university tuition fees, housing benefit and cycling proficiency lessons) and Group B (driving lessons, computer tuition, cookery lessons, roadside assistance and public transport passes) with participants viewing scenarios selected from only one Medical and one Nonmedical group in each cases. The control scenarios were always static across participants: quantity of chocolate versus carrot cakes, weight of two parcels, goals scored by a football team, size of two office buildings, number of students in two lecture classes.

Content for Medical scenarios was based on similar scenarios used in Anand and Walloo (2000). Content for Nonmedical scenarios were selected to include interventions which added to overall quality of life while not directly impacting on health and longevity (e.g., having a full driving license leads to increased social mobility). The scenarios were also subject to a pilot study in which participants were asked to specify the division of resources they viewed as fair for a variety of scenarios. Control scenarios were chosen to reflect familiar everyday constructs not related to moral judgments in any way. Examples of screens presented to participants in the scanner are shown in Figure 1.

Each slide differed in the amount of resources allocated to the groups and resource allocation was either (a) in favor of 'high' QALY groups (70/30 split or 60/40 split), (b) was equal (50/ 50 ), or (c) in favor of 'low' QALY groups (40/60, 30/70). Participants were told that the number of people falling into each category (e.g., old v young) was equal. Decisions in favor of 'high' QALY groups were therefore considered 'utilitarian' and decisions in favor of equal splits or 'low' QALY groups were considered 'nonutilitarian.'

During the task the images displayed to participants were selected from one of five stimulus sets. The sets differed in that the same split (i.e., 70/30) was never combined with the same scenario (i.e., cancer treatment)

\footnotetext{
${ }^{1}$ Instruction text read by these participants was as follows: "Utilitarianism is the philosophical perspective that moral/ethical decisions should be made with the aim of maximizing the greatest good or 'utility' to the greatest number of people. This approach is often applied to decisions in health care provision such that those that are likely to gain the most from, for example, a new drug treatment are given priority. Calculations can be based on so called quality adjusted life years (QALYs), which calculate the likely life expectancy of an individual multiplied by their quality of life. For example, someone who is married should be prioritized for treatment as on average they have a better quality of life and live longer. Similarly, those who have children, younger people and people who are financially better off would have a higher QALY score than those without children, older people or less well off individuals."
} 


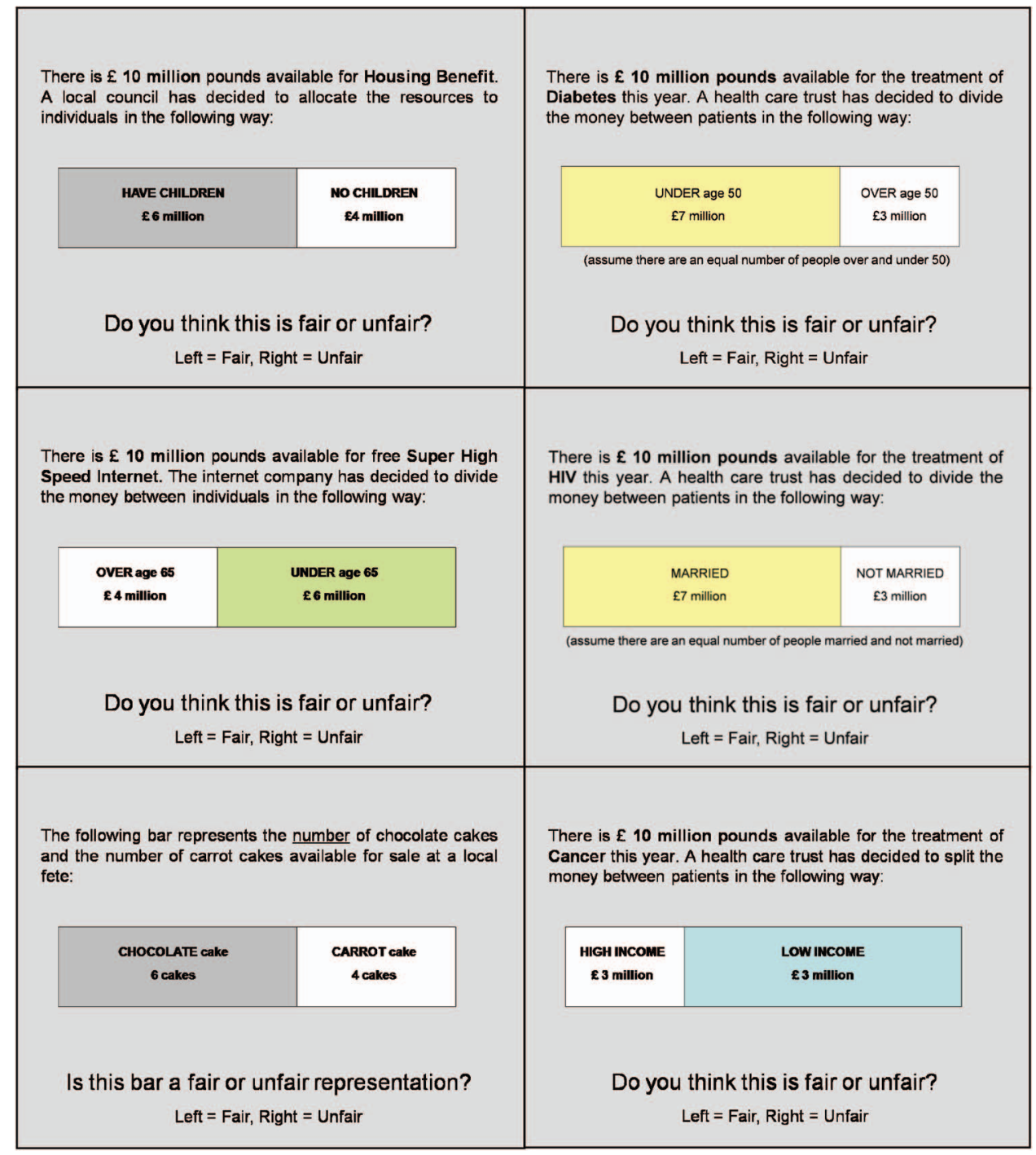

Figure 1. Examples of Control, Medical, and Nonmedical choice scenarios presented to participants. Three QALY relevant medical scenarios are presented in the right panel of the figure. Two QALY relevant Nonmedical scenarios are presented in the top two left panels of the figure. An example of a control scenario is presented in the bottom left panel. See the online article for the color version of this figure.

across sets. Within these sets the presentation of the stimulus slides were randomized across participants.

\section{Design and Procedure}

A $2 \times 5 \times 2$ mixed factorial design was employed. The repeated measures independent variables were Distribution of resources or 'splits' which had five levels, 70/30, 60/40, 50/ 50, 40/60, 30/70, and Scenario type, which had two levels, Medical or Nonmedical, with a between-group factor of Participant Instructions (whether or not instructions about QALY maximization were given to participants). The dependent variables measured were ethical standpoint (i.e., was the fair allocation judged to be 
the utilitarian distribution or the nonutilitarian distribution) and the pattern of neural activity observed in the brain via the measurement of fMRI/Blood Oxygenation Level Dependent (BOLD) signal response.

Informed consent was sought from each participant who also completed a safety checklist and received a set of written instructions for the task and screen shot of an example scenario. Participants lay face up in the scanner with cushions placed around their head to improve stability. Participants held a two-button response pad in their right hand and were instructed to press the left button if they felt the allocation of resources in each scenario was 'fair' or the right button if they felt it was 'unfair.' Between each screen a fixation cross was presented. After the completion of the fMRI session, participants read a debrief form explaining the rationale and background to the study.

\section{fMRI Data Acquisition}

A total of 455 Whole Brain 3D Echo Planar images were acquired as the participant performed the task using the Phillips 1.5T Gyroscan whole body Intera scanner at the Exeter MRI Research Centre, University of Exeter, U.K. A T2*-weighted echo planar sequence (EPI) was used with the following parameters: $\mathrm{TR}=3000 \mathrm{~ms}, \mathrm{TE}=45 \mathrm{~ms}$, flip angle $=90$ degrees, 35 transverse slices, resolution $=3$ $\mathrm{mm} \times 3 \mathrm{~mm} \times 3 \mathrm{~mm}$. FOV $=240 \mathrm{~mm}$ ascending acquisition.

\section{fMRI Preprocessing and Analysis}

Spatial preprocessing and analysis were performed using SPM-8 (http://www.fil.ion.ucl.ac .uk/spm/). Preprocessing of the data included slice-timing correction (resliced to mean image, interpolation 4th degree B spline), volume registration, realignment to first scan image (the 6 realignment parameter variables being added as nuisance covariates in the 1 st level statistical model), normalization to EPI template image, smoothing (using a 6-mm FWHM kernel, i.e., twice the voxel dimensions), and high pass filtering (SPM default of 128 second cut-off period used).

Each participant's fMRI data was analyzed using a statistical model comprising a series of regressors derived by convolving task event on- sets and durations with a canonical hemodynamic response function. The GLM approach was used to assess correlations between the measured BOLD response and regressors of interest. The 1st level statistical model included regressors corresponding to the onset of the choice screen for different resource splits for Medical, Nonmedical, and Control scenarios, such that for each subject, 12 different event regressors were represented in the first level statistical model corresponding to the following:

Medical Scenarios with 70/30 resource split, Medical-60/40, Medical-50/50, Medical-40/60, Medical-30/70 Nonmedical-70/30, Nonmedical60/40, Nonmedical-50/50, Nonmedical-40/60, Nonmedical-30/70, Control Fair Representation of Split, Control Unfair Representation Split.

One-sample $t$ tests on the statistical regressors of interest were generated for each participant and entered in the 2nd level ("random effects") analysis to look for statistically significant patterns of activation consistent across participants. Decision period activity for participants was then analyzed using a $2 \times 2 \times 5$ factorial model with (Medical/Nonmedical), participant instruction (received/did not receive briefing about QALYs) and split type (70/30, $60 / 40,50 / 50,40 / 60,30 / 70$ ) as factors. Statistical contrasts, comparing equal versus unequal splits of resources were constructed by applying positive and negative $t$-contrast weightings to the relevant regressors within the 2nd level SPM model. Because of the previously reported finding of a bias toward judging equality as "fair" in health care rationing (Anand \& Walloo, 2000; see also behavioral analysis below), the reported analysis focused on main and interaction effects of Equal versus Unequal split scenarios, equating to the comparison between regressors representing trials where participants viewed 50/50 split scenarios compared to all other resource splits (70/30, 60/40, 40/60, 30,70). Parametric modeling of the different levels of trial split was not used because it was considered that the key comparison of interest was between equality and nonequality rather that the relative quantitative split across scenarios.

Two participants were excluded from the 2 nd level fMRI factorial analysis described below because of a large amount of missing behavioral 
data where no button press response was given (one participant) and another for providing an idiosyncratic response pattern (judging all scenarios as "fair"). Second level factorial analysis was therefore based on results from 28 subjects. Results are reported at $p<.001$ threshold uncorrected for multiple comparisons.

Estimated Brodmann area (BA) reference numbers and anatomical labels were generated for each reported activation focus by first converting MNI coordinates to Talaraich coordinates (Talairach \& Tournoux, 1988) according to the "mni2tal" transformation (www.mrc-cbu .cam.ac.uk/Imaging/mnispace.html). These coordinates were then entered into the Talaraich Daemon database client software (Lancaster et al., 2000) to generate relevant anatomical labels.

\section{Results}

\section{Behavioral Data}

Nonutilitarian versus utilitarian decision makers. Using a median split, participants were classified as either 'strong' nonutilitarian or 'weak' nonutilitarian decision makers based upon their responses to the scenarios (consistent with previous behavioral investigations all subjects showed some bias toward nonutilitarianism as evidenced by a preference for objective equality when judging fairness). Analysis of behavioral choices showed that more subjects held a strong nonutilitarian "deontological" position in the subject group who were not given information about the application of QALY maximization to resource allocation prior to the experiment (10/15 subjects holding a strong antiutilitarian view when not given information about QALY versus 6/15 subjects who were given information about QALY). However, a Pearson Chi Square showed that there was no significant relationship between information about QALYs given to participants during the consent procedure and their ethical standpoint, $\chi^{2}(1), N=28, p=.274$.

Fairness decision behavior. The proportion of scenarios judged as "unfair" differed significantly across Medical and Nonmedical scenarios, $F(1,27)=5.72, p<.05$, with participants judging significantly more Medical scenarios as unfair $(67 \%)$ than Nonmedical scenarios $(62 \%)$. Participants also judged signifi- cantly more $50-50$ or 'equal splits' as fair than any of the utilitarian or nonutilitarian unequal splits, $F(4,108)=47.25, p<.001$. The judgments of fairness differed across Medical and Nonmedical scenarios according to the resource allocation split, $F(4,108)=7.86, p<.001$. This interaction effect indicated that fairness was judged differently across medical and nonmedical scenarios, $F(1,27)=23.80, p<.001$ with participants showing a significantly greater tendency to judge unequal splits as unfair in Medical compared with Nonmedical scenarios (see Figure 2).

Reaction times. A mixed ANOVA $(2 \times$ $5 \times 2$ ) with ethical view (Weak versus Strong Nonutilitarian) as a between subjects factor was used to analyze the relationship between ethical view, allocation of resources ('splits'), and time taken to process the decision (RT). Participants were classified into 'weak' or 'strong' nonutilitarian positions using a median split based on their percentage of fair/unfair judgments to decisions with utilitarian weighted resource allocation (see above). No significant main or interaction effects were observed although there was a trend toward a difference between participants RTs in Medical scenarios, $F(1,28)=$ $3.48, p=.074$. There was also no significant main effect based upon ethical view, $F(1,25)$, $p=.94, n s$, or interaction between ethical view and split $(70-30,60-40,50-50,40-6-, 30-$ 70).

A further 2-way ANOVA directly compared RTs for scenarios of equality with all those depicting unequal splits. For this analysis the difference between Medical and Nonmedical scenarios did reach significance, $F(1,28)=$ $5.91, p=.021$; Means for Medical $=8871 \pm$ $312 \mathrm{~ms}$ and Nonmedical $=9359 \pm 345 \mathrm{~ms}$, but there was no difference between Equal and Unequal scenarios overall, $F(1,28)=$ 0.39 ; Means for Equal $=8945 \pm 403 \mathrm{~ms}$ and Unequal: $9184 \pm 314 \mathrm{~ms}$, or interaction effect between Scenario type and Split, $F(1,28)=$ 0.20 .

Finally, we also carried out an analysis of RTs by subject choice (Fair/Unfair). A 2-way ANOVA with Choice and Scenario type as factor showed no significant main effect of Choice, $F(1,27)=1.55$, or Scenario, $F(1,27)=1.45$, and no significant interaction between the two factors, $F(1,27)=1.51$. 


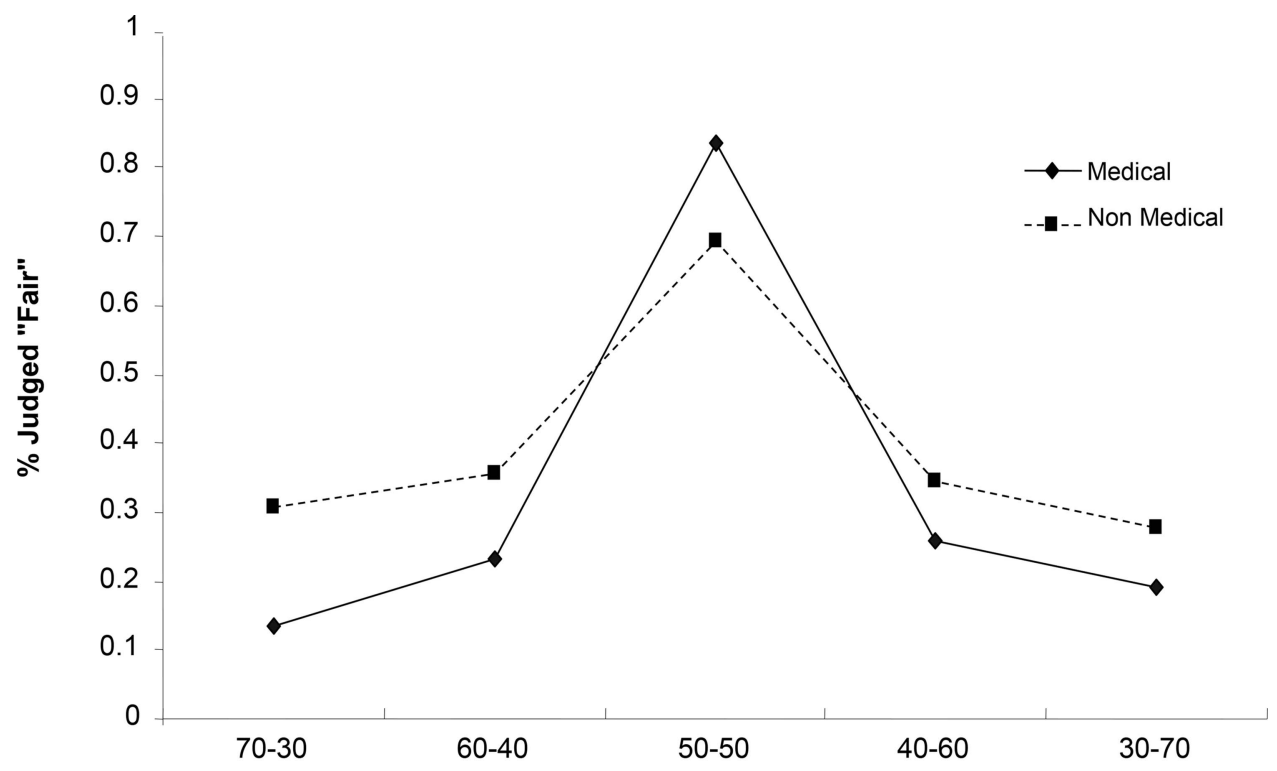

Split of resource between group

Figure 2. Proportion of scenarios judged fair versus unfair for the two scenario types (Medical/Nonmedical) plotted against resource allocation split. 70-30 splits represent those judged to be more utilitarian based on QALY factors, with 30-70 splits representing a bias in favor of groups who would be expected to have lower QALYs.

\section{fMRI Data}

Equal versus unequal splits (main effect). Comparison of brain activity during presentation of scenarios of equality - 50/50 splitsversus inequality (all other splits) revealed increased activity for equal splits within the angular gyrus (BA39), parietal lobe (BA7), middle temporal gyrus (BA19), superior frontal gyrus (BA8), precentral gyrus (BA4), and the

T1, F3 head of caudate nucleus (Table 1; Figure 3A). The bilateral head of caudate locus also included some "activated" voxels overlapping the adjacent frontal ventricle on the standard MNI template image (see Figure 4). These are likely to be artifacts of the standard smoothing and normalization procedures used to process fMRI data (see discussion). No voxels were activated during presentation of scenarios with unequal as compared to equal splits overall.

Medical versus Nonmedical decision making (main effect). Comparison of brain activity between scenarios types (Medical/Nonmed- ical scenarios) showed no significantly activated voxels.

Equality versus inequality in Medical versus Nonmedical scenarios (interaction effect). The interaction effect comparing the effect of equality (vs. inequality) in Medical and Nonmedical scenarios revealed foci of significantly increased activation in the right inferior frontal gyrus (BA9/BA47), right insula (BA13), and inferior parietal lobe (BA40; exact coordinates are given in Table 1 and Figure 3B). To further understand this interaction, we applied contrast weights to either the equal or unequal split conditions separately, for both Medical and Nonmedical problems. We also plotted the contrast estimates for each of the component model regressors in the interaction. Consistent with the differences observed in the behavioral data, this revealed that the source of the interaction effect appeared to be a larger difference in activity between Equal and Unequal splits for Medical as compared with Nonmedical scenarios (Figure 5A). 
Table 1

Table of Peak Activation Coordinates (MNI Coordinates) and Anatomical Labels for the Statistical Contrasts of fMRI/BOLD Signal Response Described in the Text

\begin{tabular}{|c|c|c|c|c|}
\hline \multirow[b]{2}{*}{ Contrast/region (Brodmann areas) } & \multirow[b]{2}{*}{$Z$ score } & \multicolumn{3}{|c|}{ MNI coordinates } \\
\hline & & $x$ & $y$ & $\mathrm{z}$ \\
\hline \multicolumn{5}{|l|}{ Main effect equal $>$ unequal splits } \\
\hline Angular gyrus (BA39) & 6.60 & 45 & -58 & 10 \\
\hline Superior parietal lobe (BA7) & 6.52 & -18 & -79 & 34 \\
\hline Middle temporal gyrus (BA19) & 6.13 & 39 & -79 & 16 \\
\hline \multirow[t]{2}{*}{ Bilateral head of caudate } & 5.20 & 6 & 11 & 13 \\
\hline & 5.20 & -6 & 17 & 7 \\
\hline Pre-central gyrus (BA4) & 4.66 & 21 & -25 & 70 \\
\hline Superior frontal gyrus (BA8) & 4.58 & 12 & 44 & 52 \\
\hline Superior parietal lobule (BA7) & 4.26 & 21 & -55 & 55 \\
\hline Pre-central gyrus (BA6) & 3.85 & -51 & -4 & 46 \\
\hline Superior temporal gyrus (BA41) & 3.12 & 42 & -31 & 16 \\
\hline \multicolumn{5}{|c|}{$\begin{array}{l}\text { Interaction effect between unequal }>\text { equal contrast for nonmedical } \\
\text { versus medical scenarios }\end{array}$} \\
\hline Inferior frontal gyrus (BA9/47) & 5.01 & 45 & 8 & 22 \\
\hline Insula (BA13) & 4.73 & 39 & 2 & 4 \\
\hline Inferior parietal lobe (BA40) & 4.09 & 60 & -37 & 37 \\
\hline \multicolumn{5}{|c|}{$\begin{array}{l}\text { Interaction effect between unequal }>\text { equal and participant instruction } \\
\text { group (participants briefed about QALYs or not) }\end{array}$} \\
\hline Inferior frontal gyrus (BA47) & 3.40 & 39 & 23 & -17 \\
\hline Middle frontal gyrus (BA11) & 3.15 & -30 & 38 & -2 \\
\hline \multicolumn{5}{|c|}{ Comparison of activity between experimental versus control trials overall } \\
\hline Limbic lobe/posterior cingulate (BA30) & 5.72 & -21 & -55 & 10 \\
\hline Caudate & 5.75 & -21 & -37 & 16 \\
\hline Parietal lobe (BA7) & 5.81 & -9 & -49 & 52 \\
\hline Post-central gyrus (BA47) & 4.91 & -48 & -16 & 22 \\
\hline Superior frontal gyrus (BA6) & 4.47 & -18 & 11 & 52 \\
\hline Medial frontal gyrus (BA9) & 3.93 & 27 & 41 & 43 \\
\hline Pre-central gyrus (BA4) & 3.66 & 48 & -10 & 52 \\
\hline
\end{tabular}

The comparison of activity when viewing trials depicting equality with inequality inevitably compared experimental conditions which contained unequal trial numbers (as four versions of each scenario presented different unequal resource splits depicted scenarios whereas only one version depicted equality). We therefore repeated the interaction analysis utilizing only trials depicting equality and those depicting the most "utilitarian" split of resources, which favored the group which might be seen to have most to gain in terms of QALYs (e.g., 70:30 in favor of young relative to old people), thus ensuring an equal number of measurements contributed to the analysis in each participant. This supplementary analysis revealed a near identical pattern of activity to that reported in the main interaction analysis including the foci in the right inferior frontal gyrus and insula cortex.
Subject instruction (main effect). Comparison of brain activity associated with subject instruction (those who had received instructions about QALY maximization vs. those whom had not) showed no significantly activated voxels.

Equality and instruction (interaction effect). The interaction effect comparing the effect of equality (see above) for the two instruction groups showed significant differences in activation to the effect of scenario equality dependent on whether or not participants received instructions concerning principles of QALY maximization. Regions activated included the inferior frontal gyrus (BA47) and middle frontal gyrus (BA11). Exact coordinates are given in Table 1 and illustrated in Figure 3C. As with the interaction between scenario type and equality (see above) we applied contrast weights to either the Equal or Unequal split conditions separately for Medical and Nonmed- 
A
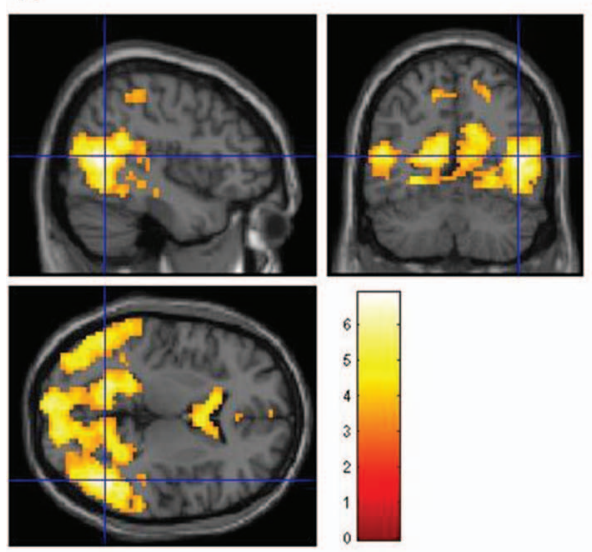

C
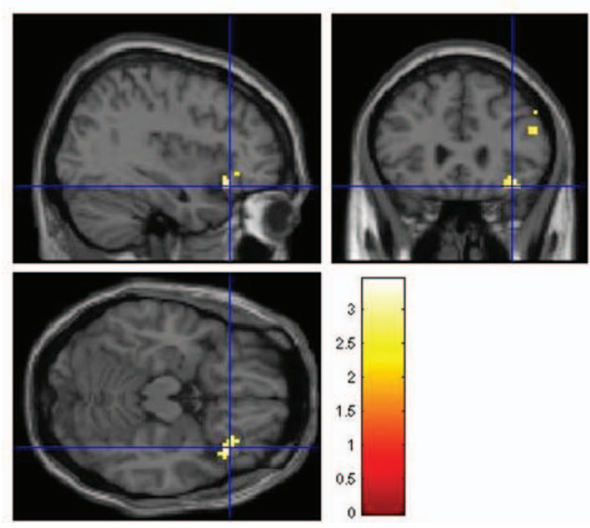

B
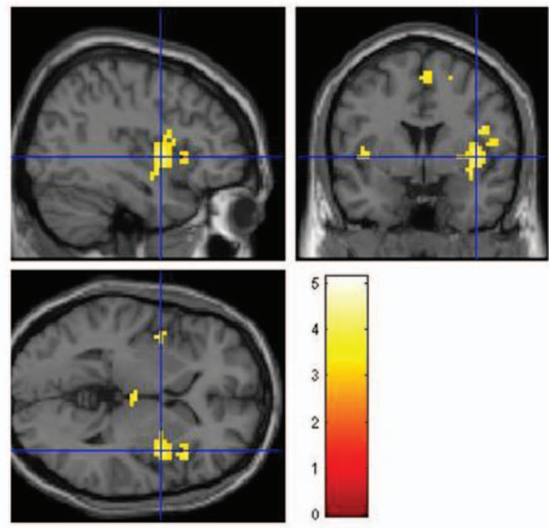

Figure 3. (A) Significant regions of activity for scenarios describing equal relative to unequal resource splits (main effect of equality; color bar shows $t$ statistic for all images). (B) Increased activity observed in inferior frontal cortex/insula cortex region to unequal scenarios for Nonmedical relative to Medical scenarios (Equality by Scenario type interaction effect). (C) Area of inferior frontal cortex showing increased activation when viewing unequal scenarios (Medical and Nonmedical) for participants given instructions explaining principles of QALY/Utilitarian decision making relative to those who were not (Interaction effect between equality and Instruction group). Color Bars indicate $t$ statistic for each contrast displayed. See the online article for the color version of this figure.

ical problems and plotted the contrast estimates for each of the component model regressors in the interaction. This showed that activity in the inferior frontal gyrus and middle frontal gyrus was increased for scenarios of inequality in the Instructed subject group (Figure 5B). As for the interaction effect by scenario type above, this analysis was repeated including only trials depicting either equality or 70:30 splits in favor of high QALY gain groups. This showed a similar locus of activity in the right inferior frontal gyrus as well additional loci within the left inferior and anterior cingulate gyrus (BA32).

Comparison by ethical view. A separate statistical model comparison of brain activity showed no significant main or interaction effect on brain activation between those who were classified as Weak versus Strong Nonutilitarian decision makers (see Behavioral Data above).

Experimental versus control trial comparison. The main effect comparison of Experimental versus Control trials overall revealed 

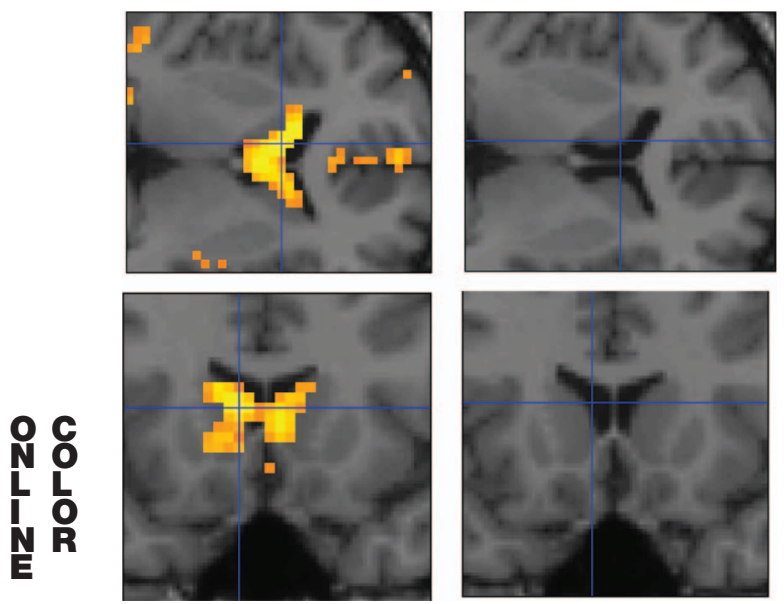

Figure 4. Zoomed axial and coronal views of a statistical parametric map overlay image (left), alongside the same sectional views of a T1 MNI standardized brain image without the overlay (right) for the contrast of scenarios depicting Equality $>$ Inequality. Voxels fulfilling $p<.001$ uncorrected for multiple comparisons statistical threshold are shown located in the grey matter of the head of caudate nucleus bilaterally. See the online article for the color version of this figure.

widespread activation for both comparisons of scenarios depicting equal and unequal splits of resources or commodities, including enhanced activity for the experimental condition in regions including the limbic lobe and posterior cingulate (BA30), parietal lobe (BA7), temporal lobe, and frontal lobe (BA6, BA4 and BA9; see Table 1).

\section{Discussion}

\section{Key Findings}

The key finding of the current study was that under conditions for which participants more often judged unequal splits of resources as "fair," relative increases in activity were seen in brain areas linked to cognitive control processes including the DLPFC, inferior frontal cortex (IFC), and anterior cingulate gyrus. Specifically, a greater difference in activation between unequal and equal splits of resources was seen in the right IFC and DLPFC (BA47 \& BA9) for medical scenarios, compared with nonmedical ones. Inspection of the contrast estimates for this interaction effect suggests a relative deactivation in these regions during viewing of med- ical scenarios depicting uneven allocation of resources to different patient groups, but no difference in BOLD signal in this region dependent on resource split for Nonmedical scenarios (Figure 5A).

\section{Implications for Accounts of Moral Decision Making}

One interpretation of these results is that making utilitarian judgments recruits brain regions in the frontal cortex associated with cognitive control functions and that there may be more sustained engagement of these regions during consideration of Nonmedical compared with Medical scenarios and for participants who received prior instruction about QALY based decision making. An explanation for this observed interaction effect might be that cognitive control systems are disrupted by presentation of scenarios perceived to breach ethical and social norms of equality of access to health care. This is consistent with the accompanying behavioral data, which replicated the previously reported bias for individuals to judge only equal resource splits as fair, particularly in medical scenarios. In these contexts, cognitive control may be less engaged and participants might be more likely, as a result, to judge unequal allocations of resources as unfair.

The right IFC region highlighted in the present study has been strongly implicated in cognitive control processes. The role of the IFC in cognitive control has been investigated predominantly using the Go/No-Go paradigm to test response inhibition where the index of inhibitory control is the number of errors made when an individual makes a button press response to a trial they should not (Aron, Robbins, \& Poldrack, 2004). The IFC becomes activated during these trials of response inhibition (Konishi et al., 1999; Liddle, Kiehl, \& Smith, 2001; Menon, Adl3eman, White, Glover, \& Reiss, 2001; Rubia, Smith, Brammer, \& Taylor, 2003). Moreover, damage to the right inferior frontal gyrus leads to disruption in stop-signal cued response inhibition (Aron, Fletcher, Bullmore, Sahakian, \& Robbins, 2003). Maturation of this area is also related to response inhibition, with a significant relationship between speed of inhibition and age (Tamm, Menon, \& Reiss, 2002). The inhibitory control functions of this region have also been shown to extend across motor response 


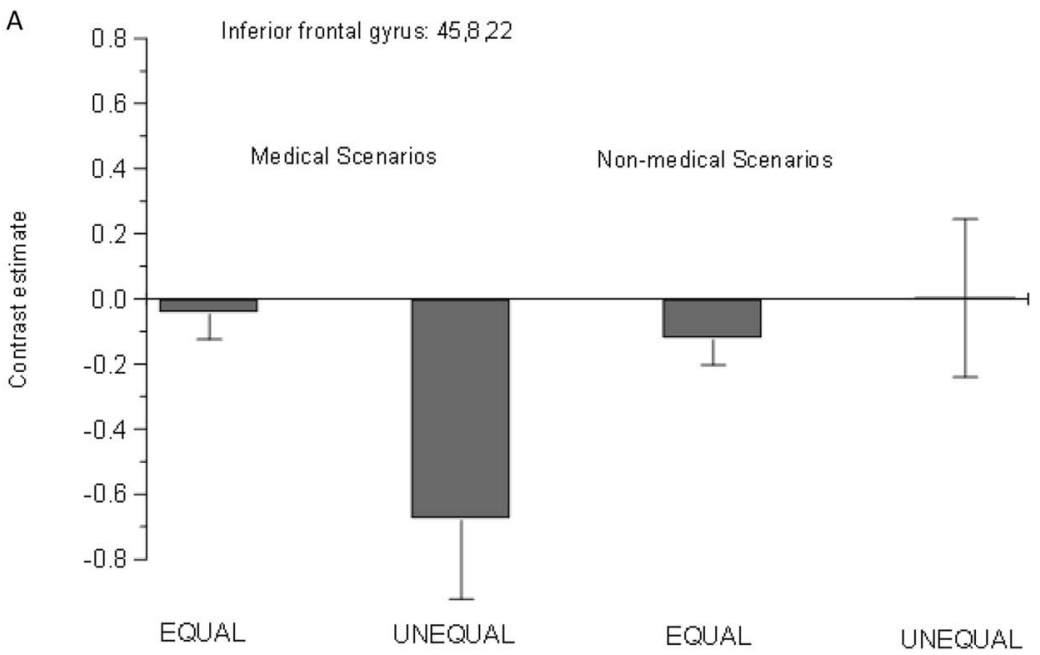

B Inferior frontal gyrus: $39,23,-17$

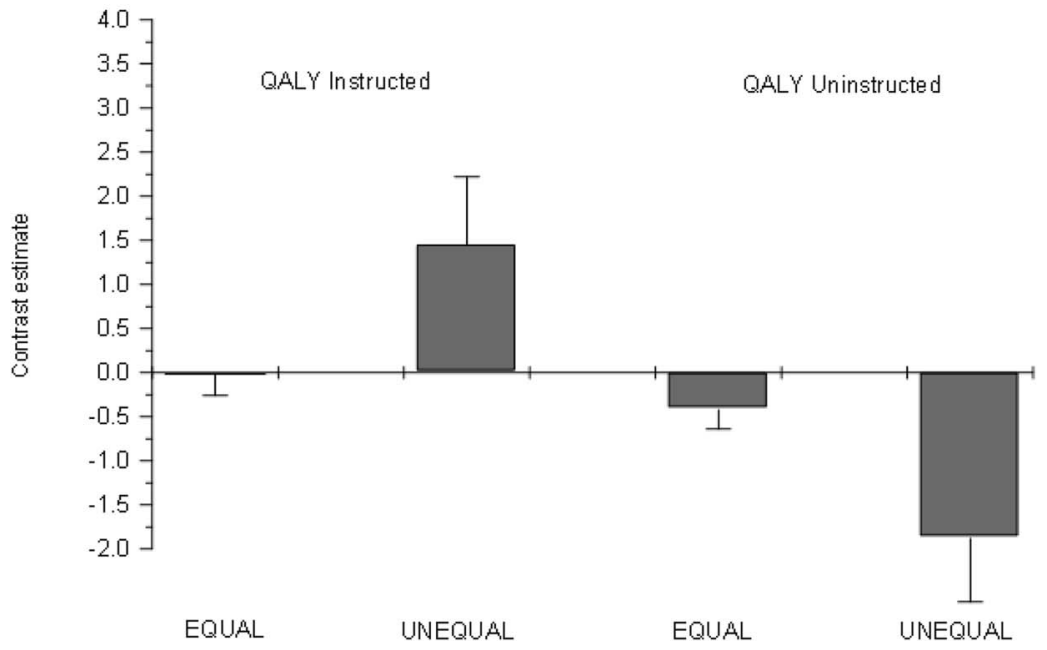

Figure 5. Contrast estimate plots for peak activation voxels in the inferior frontal gyrus for the interaction effect contrasts shown in Figure 3 and Table 1 (MNI coordinates shown). Vertical scale approximates to mean Beta coefficients fitted to the BOLD signal data for each trial/event regressor within the first level SPM analysis. (A) Contrast estimates for Medical and Nonmedical scenarios depicting Equal and Unequal splits of resources. (B) Contrast estimates for participants who were instructed or uninstructed in QALY decision making.

modalities (Hodgson et al., 2007; Aron et al., 2004).

The IFC has previously been shown to play a role in social cognition and has been implicated in the ability to predict the intentions of others (Brunet, Sarfarti, Hardy-Bayle, \& Decety, 2000). Using TMS to induce temporary lesions, it has also been shown that the left inferior frontal gyrus plays a role in social perception (Keuken et al., 2011). There are direct connections between the IFC and the medial prefrontal cortex, an area involved in self-other representations and has been suggested that this pathway facilitates integration of information necessary 
for self-other representations (Uddin, Iacoboni, Lange, \& Keenan, 2007). Further support for the role of the IFC in social cognition has been shown by the involvement of this region in more removed forms of social cognition such as the watching of movie clips depicting everyday social interactions (Iacoboni et al., 2004) or during responses to incongruent cues in social stimuli for example, press left when a face showed a rightward directed gaze (Schilbach et al., 2011).

The current results are only partially consistent with previously outlined "dual process" accounts of moral judgment and decision making (Greene, 2007). Earlier studies have emphasized the anterior cingulate region and the DLPFC as implementing cognitive control over prepotent emotional response (Greene et al., 2004). The current results indicate that the relative contribution of the IFC region to decision making processes also needs to be considered in such contexts. The behavioral bias of participants to judge only objective equality as fair also suggests that learned social rules and norms (i.e., "Rawlsian" ideas of fairness) may be important in shaping peoples judgment of health care resource rationing as are emotional systems. As well as suppressing a conflicting emotional response, cognitive control systems may also need to be engaged to suppress a neural response to violations of prevalent social values and norms in such scenarios (Hodgson, Guala, Miller, Summers, 2012) to implement more utilitarian decision making frameworks.

The activation observed during judgments of fairness in the IFC region was centered on BA47 but also extended rostrally and dorsally into other lateral frontal areas including BA11 and BA9 (i.e., DLPFC). Activation was also observed in the adjacent anterior insula during the task for the comparison of Medical scenarios compared with Nonmedical scenarios. It is worth noting that the insula has been previously implicated in social decision making and particularly in mediating the emotion of social disgust (Sanfey et al., 2003; Phillips et al., 1997; Adolphs, Tranel, \& Damasio, 2003; Calder, Keane, Manes, Antoun, \& Young, 2000; Carr, Iacoboni, Dubeau, Mazziotta, \& Lenzi, 2003; Wright, He, Shapira, Goodman, \& Liu, 2004). Further work would be needed to characterize participants' emotional responses to confirm that disgust plays a critical role in guiding par- ticipants' responses in the current experiment. An alternative explanation is that insula activity in this context represents empathetic pain response in participants' accompanying the perceived social injustice depicted in the scenario (Gu et al., 2012). Nonetheless, the fMRI results suggest that there may be something special about the processing of medical scenarios depicting unequal resource splits which modulates activity in the insula region.

Another region that showed differential activation in response to equality versus inequality in the present study was an anterior temporal lobe region around the middle temporal gyrus (see Table 1). Other research has shown the middle temporal gyrus to be activated in social contexts for example when subjects empathize with victims of crime (Farrow et al., 2001). Other studies have implicated this region in the representation of abstract conceptual knowledge of social behaviors (Zahn et al., 2007), and it has been proposed to form part of a widespread neuronal network that supports social cognition by providing access to social concepts or rules (Ross \& Olson, 2010).

Interestingly, other research has shown that moral choices and preferences are subject to variation dependent upon the form and context within which a question is presented (Tversky \& Kahneman, 1981). Our findings indicate that the instructions given to participants about the basis of utilitarian decision making and QALYs can influence their choices and modify brain activity (Figure 3C). Nord (1995) has demonstrated "framing effects" in a person trade-off scenario in which social value is estimated by asking people to state the number of outcomes of one kind they consider equal in social value to another kind. One direction for future research in the area would be to further investigate framing effects in health care resource allocation decision making within a neuroethical perspective. Another interesting study which could further investigate the role of cognitive control and inhibitory processing would be to provide participants with a secondary cognitive task while they perform similar judgments to those used here. Previous work has indicated that providing participants with an additional cognitive load during decision making may disrupt utilitarian decision making, leading to quicker response times and reduced cognitive 
control over decision making (Greene et al., 2008).

\section{Limitations and Criticisms}

This is a pioneering but relatively small-scale fMRI study of moral choice in health care decision making. As such it is important to discuss the limitations and criticisms of the study design and analysis.

The choice of control task used can be criticized. We designed the control task to be a close match with the experimental task in terms of the visual properties of the screen and the motor action to be given by the participant (left or right button press). Other aspects of the control trials were designed to be very different from the experimental task, with participants being required to make a judgment as to whether the bar indicator matched the written description of a quantitative split of a commodity. In this respect, control trials lacked any demands on emotional and moral decision making and placed minimal cognitive demands on the participant. We acknowledge that other forms of control task could have been used, and the one used here may be considered to be too different to the experimental condition to provide a useful comparison. However, it is important to note that for trial/event related fMRI designs such as used here, the effective baseline for comparisons of activity with experimental trials is brain activity during null periods between the decision screen onsets. Most of the contrasts of interest reported reflect direct comparisons between experimental trial types which were closely matched other than in a single variable and were independent of activity elicited by the control task.

Another aspect of the task which should be noted is that participants always indicated their attribution of fairness or unfairness by pressing the left or the right response key on a button box held in their dominant (right hand) and the mapping of finger to choice was not counter balanced across participants. It is conceivable therefore that some aspects of the results might arise from activation differences associated with the movement of the index and middle finger rather than processing of the presented scenario. However, no activation differences were found in the primary motor cortex for any of the contrasts of fair versus unfair judgments and the reported analysis of activity was focused on the epoch before response execution rather than after the response. Further, the most interesting aspect of the results were interaction effects which could not be simply explained by any confounding effect of preparatory motor activity. Overall, therefore, it seems very unlikely that this minor feature of the design had an effect on the results.

The analysis of fMRI data used the general linear model (GLM) approach. Although this is the most common statistical technique for analyzing fMRI data, it has been criticized for being too rigid, with minor mis-modeling resulting in material loss of statistical power (Lindquist, 2008). These issues apply to most fMRI work at present and call either for the use of larger samples or more advanced analysis techniques (see for instance a Bayesian approach developed by Woolrich, Behrens, Beckmann, Jenkinson, \& Smith, 2004). However, a specific statistical issue affecting interpretation of the present experiment is the low number of trials presented for some conditions of interest (e.g., four medical scenarios depicting equality per participant). The number of stimulus presentations was limited to minimize the amount of time participants spent in the scanner and to ensure that participants attended to the task throughout (total task length was 28 minutes and total time in scanner per subject was around 40-45 minutes). Although it is more common practice to average fMRI data over more trials, there is no commonly accepted minimum number of trials per condition or participants for effective fMRI investigations, with some studies having successfully measured the BOLD response to single trials in single subjects (Posse et al., 2001; Mechelli, Price, Henson, \& Friston, 2003). The minimum number of trials required to measure a given BOLD response depends on the size of the effect to be measured, together with the number of participants tested and the inherent variability of the measured signal (all of which factor together to determine the statistical power of a given study).

Neuroimaging also requires that individual brain images are transformed into a standardized brain space such that data can be averaged across subjects with different brain dimensions and morphology. Spatial smoothing of signals reduces artifactual signal noise but places further constraints on the spatial accu- 
racy of the technique. These preprocessing techniques can lead to potential errors in precisely coregistering activity within specific anatomical substrates, particularly for small and closely adjacent regions. This is the most likely explanation for the artifactual "activation" of a small number of voxels in the frontal ventricles adjacent to the caudate nuclei in the present study (see Figure 4).

A different source of general concern about applying fMRI to moral and ethical situations is highlighted by Schleim and Schirmann (2011), who argue that the normative implications and uncertainties are too readily ignored or disregarded by some when discussing neuroimaging research. They propose that the findings of fMRI studies do not by themselves yield normative implications, that is, prescribe how one ought to act. It should also be noted that there is a strong "reverse inference" problem underlying interpretation of neuroimaging in such contexts. Even relatively well-evidenced claims about functions mediated by particular brain areas (e.g., IFC mediates cognitive inhibitory control) are still subject to debate and discussion within cognitive neuroscience. Brain imaging data cannot therefore necessarily be viewed as a more objective/definitive a line of enquiry as other approaches. The neuro-ethical perspective offered here cannot resolve the ongoing debates between libertarians and egalitarian theorists but offers an interesting framework within which to think about the issues (Casebeer \& Churchland, 2003; Braveman \& Gruskin, 2003; Sandel, 2009; Friesen, 2001; Leary, 1994).

Finally, it should be noted that all of our participants were young (aged between 16 and 36), native English speakers studying or working in the U.K. Therefore the behavioral decisions and accompanying neural activity in these participants might be quite different in participants with experience of different health care systems, social group/identity characteristics, and cultural values.

\section{Concluding Remarks}

This is the first study to use fMRI to investigate moral fairness decision making judgments in health care resource allocation scenarios. Consistent with previous work using purely behavioral measures (Anand \& Walloo, 2000), we found evidence for an egalitarian decision making bias in medical treatment rationing scenarios. Differences in brain activity in response to presentation of equal versus unequal splits of resources in medical and nonmedical scenarios were observed which provide indicators of the mechanism underlying the behavioral bias toward objective equality observed in participants in judgments of fairness. A particular role for the inferior frontal cortex (IFC) is suggested in overriding the bias toward judging only objectively equal resource splits as fair, whereas heightened activation of the insula may indicate a reaction of disgust toward breaches of social norms or empathetic pain evoked by perceived inequality in other social groups' access to health care.

The current study should be seen as making a small contribution to a question of very large global socioeconomic importance. As such we have taken care to list the methodological and analytical limitations and criticisms of the study. However, as an initial investigation of the biological mechanisms underlying human decision making in this area we hope the work may prompt new ways of thinking about fairness and inequality that could be value to economists and philosophers alike.

\section{References}

Adolphs, R., Tranel, D., \& Damasio, A. R. (2003). Dissociable neural systems for recognizing emotions. Brain and Cognition, 52, 61-69. http://dx .doi.org/10.1016/S0278-2626(03)00009-5

Anand, P., \& Walloo, A. (2000). Utilities versus right to publically provided goods: Arguments and evidence from health care rationing. Economica, 67, 543-577. http://dx.doi.org/10.1111/1468-0335 .00224

Appleby, J., Devlin, N., \& Parkin, D. (2007). NICE's cost effectiveness threshold. British Medical Journal, 335, 358-359. http://dx.doi.org/10.1136/bmj 39308.560069.BE

Aron, A. R., Fletcher, P. C., Bullmore, E. T., Sahakian, B. J., \& Robbins, T. W. (2003). Stop-signal inhibition disrupted by damage to right inferior frontal gyrus in humans. Nature Neuroscience, 6, 115-116. http://dx.doi.org/10.1038/nn1003

Aron, A. R., Robbins, T. W., \& Poldrack, R. A. (2004). Inhibition and the right inferior frontal cortex. Trends in Cognitive Sciences, 8, 170-177. http://dx.doi.org/10.1016/j.tics.2004.02.010

Bechara, A., \& Damasio, A. R. (2005). The somatic marker hypothesis: A neural theory of economic decision. Games and Economic Behavior, 52, 
336-372. http://dx.doi.org/10.1016/j.geb.2004.06 .010

Bentham, J. (1789). An introduction to the principles of morals and legislation. London, UK: T. Payne \& Son.

Braveman, P., \& Gruskin, S. (2003). Defining equity in health. Journal of Epidemiology and Community Health, 57, 254-258. http://dx.doi.org/ 10.1136/jech.57.4.254

Brunet, E., Sarfati, Y., Hardy-Baylé, M. C., \& Decety, J. (2000). A PET investigation of the attribution of intentions with a nonverbal task. NeuroImage, 11, 157-166. http://dx.doi.org/10.1006/nimg .1999 .0525

Calder, A. J., Keane, J., Manes, F., Antoun, N., \& Young, A. W. (2000). Impaired recognition and experience of disgust following brain injury. $\mathrm{Na}$ ture Neuroscience, 3, 1077-1078. http://dx.doi .org/10.1038/80586

Carr, L., Iacoboni, M., Dubeau, M. C., Mazziotta, J. C., \& Lenzi, G. L. (2003). Neural mechanisms of empathy in humans: A relay from neural systems for imitation to limbic areas. PNAS Proceedings of the National Academy of Sciences of the United States of America, 100, 5497-5502. http://dx.doi .org/10.1073/pnas.0935845100

Casebeer, D. W., \& Churchland, P. S. (2003). The neural mechanisms of moral cognition: A multiple-aspect approach to moral judgment and decision-making. Biology and Philosophy, 18, 169194. http://dx.doi.org/10.1023/A:1023380907603

Ciaramelli, E., Muccioli, M., Làdavas, E., \& di Pellegrino, G. (2007). Selective deficit in personal moral judgment following damage to ventromedial prefrontal cortex. Social Cognitive and Affective Neuroscience, 2, 84-92. http://dx.doi.org/10.1093/ scan/nsm001

Cubbon, J. (1991). The principle of QALY maximisation as the basis for allocating health care resources. Journal of Medical Ethics, 17, 181-184. http://dx.doi.org/10.1136/jme.17.4.181

Farrow, T. F., Zheng, Y., Wilkinson, I. D., Spence, S. A., Deakin, J. F., Tarrier, N., . . . Woodruff, P. W. (2001). Investigating the functional anatomy of empathy and forgiveness. Neuroreport, 12, 2433-2438. http://dx.doi.org/10.1097/00001756200108080-00029

Friesen, T. (2001). The right to health care. Health Law Journal, 9, 205-222.

Greene, J. D. (2007). Why are VMPFC patients more utilitarian? A dual-process theory of moral judgment explains. Trends in Cognitive Sciences, 11, 322-323. http://dx.doi.org/10.1016/j.tics.2007.06 .004

Greene, J. D., Morelli, S. A., Lowenberg, K., Nystrom, L. E., \& Cohen, J. D. (2008). Cognitive load selectively interferes with utilitarian moral judg- ment. Cognition, 107, 1144-1154. http://dx.doi .org/10.1016/j.cognition.2007.11.004

Greene, J. D., Nystrom, L. E., Engell, A. D., Darley, J. M., \& Cohen, J. D. (2004). The neural bases of cognitive conflict and control in moral judgment. Neuron, 44, 389-400. http://dx.doi.org/10.1016/j .neuron.2004.09.027

Gu, X., Gao, Z., Wang, X., Liu, X., Knight, R. T., Hof, P. R., \& Fan, J. (2012). Anterior insular cortex is necessary for empathetic pain perception. Brain: A Journal of Neurology, 135, 2726-2735. http://dx.doi.org/10.1093/brain/aws199

Haidt, J., Rozin, P., McCauley, C., \& Imada, S. (1997). Body, psyche, and culture: The relationship between disgust and morality. Psychology and Developing Societies, 9, 107-131. http://dx .doi.org/10.1177/097133369700900105

Harris, J. (1987). QALYfying the value of life. Journal of Medical Ethics, 13, 117-123. http://dx.doi .org/10.1136/jme.13.3.117

Harris, J. (1995). Double jeopardy and the veil of ignorance-A reply. Journal of Medical Ethics, 21, 151-157. http://dx.doi.org/10.1136/jme.21.3 .151

Harris, J. (2005). It's not NICE to discriminate. Journal of Medical Ethics, 31, 373-375. http://dx.doi .org/10.1136/jme.2005.012906

Hodgson, T., Chamberlain, M., Parris, B., James, M., Gutowski, N., Husain, M., \& Kennard, C. (2007). The role of the ventrolateral frontal cortex in inhibitory oculomotor control. Brain: A Journal of Neurology, 130, 1525-1537. http://dx.doi.org/ 10.1093/brain/awm064

Hodgson, T. L., Guala, F., Miller, T., \& Summers, I. (2012). Limbic and prefrontal activity during conformity and violation of norms in a coordination game. Journal of Neuroscience, Psychology, and Economics, 5, 1-17. http://dx.doi.org/10.1037/ a0026584

Iacoboni, M., Lieberman, M. D., Knowlton, B. J., Molnar-Szakacs, I., Moritz, M., Throop, C. J., \& Fiske, A. P. (2004). Watching social interactions produces dorsomedial prefrontal and medial parietal BOLD fMRI signal increases compared to a resting baseline. NeuroImage, 21, 1167-1173. http://dx.doi.org/10.1016/j.neuroimage.2003.11 .013

Keuken, M. C., Hardie, A., Dorn, B. T., Dev, S., Paulus, M. P., Jonas, K. J., . . Pineda, J. A. (2011). The role of the left inferior frontal gyrus in social perception: An rTMS study. Brain Research, 1383, 196-205. http://dx.doi.org/10.1016/j .brainres.2011.01.073

Koenigs, M., Young, L., Adolphs, R., Tranel, D., Cushman, F., Hauser, M., \& Damasio, A. (2007). Damage to the prefrontal cortex increases utilitarian moral judgements. Nature, 446, 908-911. http://dx.doi.org/10.1038/nature05631 
Konishi, S., Nakajima, K., Uchida, I., Kikyo, H., Kameyama, M., \& Miyashita, Y. (1999). Common inhibitory mechanism in human inferior prefrontal cortex revealed by event-related functional MRI. Brain: A Journal of Neurology, 122, 981-991. http://dx.doi.org/10.1093/brain/122.5.981

Lancaster, J. L., Woldorff, M. G., Parsons, L. M., Liotti, M., Freitas, C. S., Rainey, L., . . Fox, P. T. (2000). Automated Talairach atlas labels for functional brain mapping. Human Brain Mapping, 10, 120-131. http://dx.doi.org/10.1002/1097-0193(200007)10: $3<120::$ AID-HBM30>3.0.CO;2-8

Leary, V. A. (1994). Defining the right to health care. In A. R. Chapman (Ed.), Health care reform: A human rights approach. Washington, DC: Georgetown University Press.

Liddle, P. F., Kiehl, K. A., \& Smith, A. M. (2001). Event-related fMRI study of response inhibition. Human Brain Mapping, 12, 100-109. http://dx.doi .org/10.1002/1097-0193(200102)12:2<100::AIDHBM1007>3.0.CO;2-6

Lindquist, M. A. (2008). The statistical analysis of fMRI data. Statistical Science, 23, 439-464. http://dx.doi.org/10.1214/09-STS282

Mechelli, A., Price, C. J., Henson, R. N. A., \& Friston, K. J. (2003). Estimating efficiency a priori: A comparison of blocked and randomized designs. NeuroImage, 18, 798-805. http://dx.doi .org/10.1016/S1053-8119(02)00040-X

Menon, V., Adleman, N. E., White, C. D., Glover, G. H., \& Reiss, A. L. (2001). Error-related brain activation during a $\mathrm{Go} / \mathrm{NoGo}$ response inhibition task. Human Brain Mapping, 12, 131-143. http:// dx.doi.org/10.1002/1097-0193(200103)12:3< 131::AID-HBM1010>3.0.CO;2-C

Moll, J., \& de Oliveira-Souza, R. (2007). Moral judgments, emotions and the utilitarian brain. Trends in Cognitive Sciences, 11, 319-321. http:// dx.doi.org/10.1016/j.tics.2007.06.001

Moll, J., de Oliveira-Souza, R., \& Eslinger, P. J. (2003). Morals and the human brain: A working model. Neuroreport, 14, 299-305. http://dx.doi .org/10.1097/00001756-200303030-00001

Moll, J., de Oliveira-Souza, R., Eslinger, P. J., Bramati, I. E., Mourão-Miranda, J., Andreiuolo, P. A., \& Pessoa, L. (2002). The neural correlates of moral sensitivity: A functional magnetic resonance imaging investigation of basic and moral emotions. The Journal of Neuroscience, 22, 27302736.

Moll, J., de Oliveira-Souza, R., Moll, F. T., Ignácio, F. A., Bramati, I. E., Caparelli-Dáquer, E. M., \& Eslinger, P. J. (2005). The moral affiliations of disgust: A functional MRI study. Cognitive and Behavioral Neurology, 18, 68-78. http://dx.doi .org/10.1097/01.wnn.0000152236.46475.a7

Moll, J., Eslinger, P. J., \& de Oliveira-Souza, R. (2001). Frontopolar and anterior temporal cortex ac- tivation in a moral judgment task: Preliminary functional MRI results in normal subjects. Arquivos de Neuro-Psiquiatria, 59, 657-664. http://dx.doi .org/10.1590/S0004-282X2001000500001

Nord, E. (1995). The person-trade-off approach to valuing health care programs. Medical Decision Making, 15, 201-208. http://dx.doi.org/10.1177/ 0272989X9501500302

Phillips, M. L., Young, A. W., Senior, C., Brammer, M., Andrew, C., Calder, A. J., . . . David, A. S. (1997). A specific neural substrate for perceiving facial expressions of disgust. Nature, 389, 495498. http://dx.doi.org/10.1038/39051

Posse, S., Binkofski, F., Schneider, F., Gembris, D., Frings, W., Habel, U., . . . Eickermann, T. (2001). A new approach to measure single-event related brain activity using real-time fMRI: Feasibility of sensory, motor, and higher cognitive tasks. Human Brain Mapping, 12, 25-41. http:// dx.doi.org/10.1002/1097-0193(200101)12: $1<25:$ :AID-HBM30>3.0.CO;2-H

Rawls, J. (1971). A theory of justice. Cambridge, MA: Harvard University Press.

Ross, L. A., \& Olson, I. R. (2010). Social cognition and the anterior temporal lobes. NeuroImage, 49, 3452-3462. http://dx.doi.org/10.1016/j.neuroimage.2009.11.012

Rubia, K., Smith, A. B., Brammer, M. J., \& Taylor, E. (2003). Right inferior prefrontal cortex mediates response inhibition while mesial prefrontal cortex is responsible for error detection. NeuroImage, 20, 351-358. http://dx.doi.org/10.1016/ S1053-8119(03)00275-1

Sandel, M. (2009). Justice: What's the right thing to do? New York, NY: Penguin.

Sanfey, A. G., Rilling, J. K., Aronson, J. A., Nystrom, L. E., \& Cohen, J. D. (2003). The neural basis of economic decision-making in the Ultimatum Game. Science, 300, 1755-1758. http://dx.doi .org/10.1126/science.1082976

Schilbach, L., Eickhoff, S. B., Cieslik, E., Shah, N. J., Fink, G. R., \& Vogeley, K. (2011). Eyes on me: An fMRI study of the effects of social gaze on action control. Social Cognitive and Affective Neuroscience, 6, 393-403. http://dx.doi.org/ $10.1093 /$ scan/nsq067

Schleim, S., \& Schirmann, S. (2011). Philosophical implications and multidisciplinary challenges of moral physiology. Trames, 15, 127-146. http://dx .doi.org/10.3176/tr.2011.2.02

Talairach, J., \& Tournoux, P. (1988). Co-planar stereotactic atlas of the human brain: 3-dimensional proportional system: An approach to cerebral imaging. New York, NY: Thieme.

Tamm, L., Menon, V., \& Reiss, A. L. (2002). Maturation of brain function associated with response inhibition. Journal of the American Academy of Child \& Adolescent Psychiatry, 41, 1231-1238. 
http://dx.doi.org/10.1097/00004583-20021000000013

Tversky, A., \& Kahneman, D. (1981). The framing of decisions and the psychology of choice. Science, 211, 453-458. http://dx.doi.org/10.1126/science .7455683

Uddin, L. Q., Iacoboni, M., Lange, C., \& Keenan, J. P. (2007). The self and social cognition: The role of cortical midline structures and mirror neurons. Trends in Cognitive Sciences, 11, 153-157. http:// dx.doi.org/10.1016/j.tics.2007.01.001

Woolrich, M. W., Behrens, T. E., Beckmann, C. F., Jenkinson, M., \& Smith, S. M. (2004). Multilevel linear modelling for FMRI group analysis using Bayesian inference. NeuroImage, 21, 1732-1747. http://dx.doi.org/10.1016/j.neuroimage.2003.12 .023

Wright, P., He, G., Shapira, N. A., Goodman, W. K., \& Liu, Y. (2004). Disgust and the insula: FMRI responses to pictures of mutilation and contamination. Neuroreport, 15, 2347-2351. http://dx.doi .org/10.1097/00001756-200410250-00009

Young, L., \& Koenigs, M. (2007). Investigating emotion in moral cognition: A review of evidence from functional neuroimaging and neuropsychology. British Medical Bulletin, 84, 69-79. http://dx.doi .org/10.1093/bmb/ldm031

Zahn, R., Moll, J., Krueger, F., Huey, E. D., Garrido, G., \& Grafman, J. (2007). Social concepts are represented in the superior anterior temporal cortex. PNAS Proceedings of the National Academy of Sciences of the United States of America, 104, 6430-6435. http://dx.doi.org/10.1073/pnas .0607061104

Received March 8, 2013

Revision received April 27, 2015

Accepted April 28, 2015 


\section{AUTHOR QUERIES}

\section{AUTHOR PLEASE ANSWER ALL QUERIES}

AQau-Please confirm the given-names and surnames are identified properly by the colors. = Given-Name, $\boldsymbol{a}=$ Surname The colors are for proofing purposes only. The colors will not appear online or in print.

AQ1-Author: This article has been lightly edited for grammar, style, and usage. Please compare against your original document and make changes on these paged proofs. If no change is required in response to a question, please write "OK as set" in the margin.

AQ2-Author: Please be sure to provide the name of the department(s) with which you and your coauthors are affiliated at your respective institutes if you have not already done so. If you are affiliated with a governmental department, business, hospital, clinic, VA center, or other nonuniversity-based institute, please provide the city and U.S. state (or the city, province, and country) in which the institute is based. Departments should be listed in the author footnote only, not the byline. If you or your coauthors have changed affiliations since the article was written, please include a separate note indicating the new department/affiliation: [author's name] is now at [affiliation].

AQ3-Author: Please review the typeset table(s) carefully against your original table(s) to verify accuracy of editing and typesetting.

AQ5-Author: Please confirm figure(s) and legend(s) as set. 